\title{
Age-dependent regulation of cell-mediated collagen turnover
}

\author{
Michael J. Podolsky, ${ }^{1,2,3}$ Christopher D. Yang, ${ }^{1}$ Carlos Lizama Valenzuela, ${ }^{1}$ Ritwik Datta, ${ }^{1}$ \\ Steven K. Huang, ${ }^{4}$ Stephen L. Nishimura, ${ }^{5}$ Sarah L. Dallas, ${ }^{6}$ Paul J. Wolters, ${ }^{3}$ \\ Claude Jourdan Le Saux, ${ }^{3}$ and Kamran Atabai ${ }^{1,2,3}$ \\ ${ }^{1}$ Cardiovascular Research Institute, ${ }^{2}$ Lung Biology Center, and ${ }^{3}$ Department of Medicine, UCSF, San Francisco, California, \\ USA. ${ }^{4}$ Department of Internal Medicine, University of Michigan Medical School, Ann Arbor, Michigan, USA. ${ }^{5}$ Department \\ of Pathology, UCSF, San Francisco, California, USA. 'Department of Oral and Craniofacial Sciences, School of Dentistry, \\ University of Missouri-Kansas City, Kansas City, Missouri, USA.
}

\begin{abstract}
Although aging represents the most important epidemiologic risk factor for fibrotic disease, the reasons for this are incompletely understood. Excess collagen deposition in tissues is the sine qua non of tissue fibrosis and can be viewed as an imbalance between collagen production and collagen degradation. Yet we still lack a detailed understanding of the changes that take place during development, maturation, and aging in extracellular matrix (ECM) dynamics. Resolution of fibrosis is impaired in aging, and this impairment may explain why age is the most important risk factor for fibrotic diseases, such as idiopathic pulmonary fibrosis. However, ECM dynamics and impaired resolution of fibrosis in aging remain understudied. Here we show that cell-mediated collagen uptake and degradation are diminished in aged animals and this finding correlates with downregulation of the collagen endocytic receptor mannose receptor, C-type $\mathbf{2}$ (Mrc2). We identify myeloid zinc finger-1 as a potentially novel transcriptional regulator of Mrc2, and both this transcription factor and Mrc2 are downregulated in multiple tissues and organisms in an age-dependent manner. Thus, cellmediated degradation of collagen is an essential process that promotes resolution of fibrosis and impairment in this process contributes to age-related fibrosis.
\end{abstract}

Conflict of interest: The authors have declared that no conflict of interest exists.

Copyright: () 2020, American Society for Clinical Investigation.

Submitted: February 26, 2020

Accepted: April 15, 2020

Published: May 21, 2020.

Reference information: /CI Insight. 2020;5(10):e137519.

https://doi.org/10.1172/jci.

insight.137519.

\section{Introduction}

Advanced age is a major risk factor for organ fibrosis, including lung fibrosis (1). Furthermore, genetic disorders of telomere maintenance that represent a form of premature aging have been highly associated with fibrotic disease, in particular pulmonary fibrosis (2). Despite these widely recognized associations, the underlying mechanism that links aging to the development of pulmonary fibrosis is incompletely understood (3). Conversely, organ fibrosis is less commonly seen in young humans or animals. It is generally accepted that many injuries which may cause scarring in an adult will instead cause scarless healing in a developing organism (4). For example, in utero surgery generally does not produce scar tissue whereas the same surgery in a child or adult does produce scarring (5)

An open question is whether the protection from fibrosis in very young organisms represents the same biology that underlies the predisposition to fibrosis in older organisms. Recent studies have shown that impaired resolution of fibrosis may be just as important in the pathogenesis of fibrotic disorders as the onset or initial inflammatory response after tissue injury. In the bleomycin model of lung fibrosis, aged mice have been shown to exhibit a failure to resolve fibrosis compared with young animals (6). Extracellular matrix (ECM) accumulation is considered a normal response to injury, but its persistence is believed to contribute to diseases such as idiopathic pulmonary fibrosis, which is known not only to persist but also to progress and remain refractory to attempts at disease reversal (7)

The mechanisms that govern resolution of fibrosis are also not fully understood. The dynamics of ECM in general, and collagen in particular during aging and fibrosis, are not completely known. In prior decades several groups evaluated lung collagen content during early time points of maturation (8-10) using radioassays and biochemical techniques. However, these studies did not provide a direct link to fibrotic 
disease or the way normal aging affects both degradative and synthetic ECM processes. In the present study we examine how aging affects ECM turnover and how this contributes to the pathogenesis of fibrosis.

\section{Results}

Collagen is a major component of ECM, including fibrotic ECM (11). We first compared the collagen content in the lungs of young (5-8 weeks of age) and mature (10-12 months of age) mice using the hydroxyproline assay and picrosirius red staining (Figure 1, A-C). Mature mice demonstrated a higher level of total collagen content compared with young mice (Figure 1A) or collagen content normalized for lung weight (Figure 1B). To determine the mechanistic basis for this increasing collagen content, the amount of type I collagen transcripts (a surrogate for collagen synthesis) was measured and found to be lower in mature mice (Figure 1, D and E). Publicly available transcriptomic data (12) corroborate an age-dependent downregulation of type I collagen transcripts, as well as type III collagen transcripts, another fibrillar collagen (Supplemental Figure 1, A-C; supplemental material available online with this article; https://doi.org/10.1172/jci.insight.137519DS1).

Because collagen content was higher in mature mice but transcript levels were lower, we reasoned that the age-associated accumulation of collagen may result from a dysregulation of collagen degradation pathways. Collagen turnover is governed by both an extracellular proteolytic pathway and an intracellular pathway of uptake and lysosomal degradation $(11,13)$, with feedback between the 2 pathways (14). To investigate the role of different genes involved in collagen turnover, a targeted quantitative PCR (Q-RTPCR) screen for many of these genes was performed (Figure 1F). This analysis revealed a significant decrease in $M m p 2$, as well as Mrc2 (which codes for the protein mannose receptor, C-type 2, also referred to as Endo180 or uPARAP), a canonical collagen endocytic receptor (15). Though Mmp2 was decreased with aging and several other MMP genes showed nonsignificant decreases with aging, several tissue inhibitor of metalloproteinase genes also showed nonsignificant decreases with aging. To investigate functionally whether the extracellular pathway was impaired with aging, we used the DQ-collagen assay, which measures the collagenolytic activity of tissue lysates by quantifying their ability to cleave a fluorescent type I collagen molecule (16). We found robust liberation of collagen fragments from lysates of both young and mature mice (Figure $1 \mathrm{G}$ ). The ability of lysates to cleave collagen was nearly completely quenched with the pan-MMP inhibitor GM6001. Taken together, these data suggested that extracellular proteolytic cleavage was intact with aging. Given that the Q-RT-PCR data and the DQ-collagen assay results did not reveal clear differences in the activity of the extracellular pathway, we focused on the cell-based collagen degradative machinery, which is known to be critically dependent on Mrc2 (17).

The level of Mrc2 expression was decreased in the lungs of mature mice compared with young mice at both the protein (Figure 1, H and I) and transcript level (Figure 1J). A time course of MRC2 protein expression in whole mouse lung showed progressive, age-dependent downregulation (Figure 1K), which was also corroborated by transcriptomic data from an outside lab (Supplemental Figure 1D) (12). Notably, mannose receptor or Mrc1 is in the same family as Mrc2 and can internalize collagen (11). However, expression of this molecule was not decreased with maturation in our initial screen, or by Western blot, or in a separate Q-RT-PCR experiment, or in another group's data (Supplemental Figure 2) (18).

MRC2 is known to be primarily expressed on stromal cells whereas MRC1 is likely the dominant collagen endocytic receptor on myeloid cells (11). We used several orthogonal techniques to identify the cell type responsible for age-dependent downregulation of Mrc2. First, a flow cytometry-based fluorescent collagen uptake assay $(19,20)$ was used to test cellular uptake of collagen in mature animals. Collagen uptake was impaired in fibroblasts from mature animals identified as CD45-PDGFRA ${ }^{+}$but not in myeloid cells identified as $\mathrm{CD} 45^{+} \mathrm{F} 4 / 80^{+}$cells (21) (Figure 2A and Supplemental Figure 3), another important cell type involved in collagen cellular uptake and degradation (11). Immunostaining of lung tissue demonstrated that MRC2 levels are decreased in many cells that costain with the PDGFRA antibody in mature lungs (Figure 2B). Furthermore, Q-RT-PCR after sorting fibroblasts or myeloid cells using the same markers showed decreased $\mathrm{Mrc2}$ in fibroblasts (Figure 2C). Finally, a flow staining approach was used to stain for MRC2 after validating specificity of this antibody in flow cytometry (Supplemental Figure 4, A and B). This showed that fibroblasts exhibited diminished surface levels of MRC2 in mature animals, but other cell types did not show a difference (Figure 2D and Supplemental Figure 4C). Collectively these data show that cell-mediated collagen uptake in fibroblasts decreases during maturation along with fibroblast-specific Mrc2 expression and whole-lung Mrc2 expression. 
A

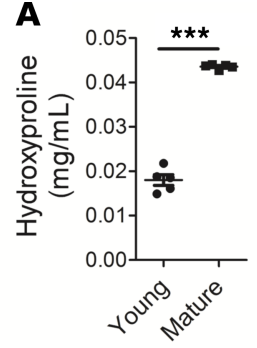

B

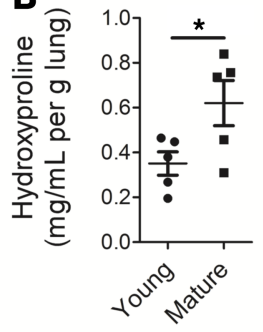

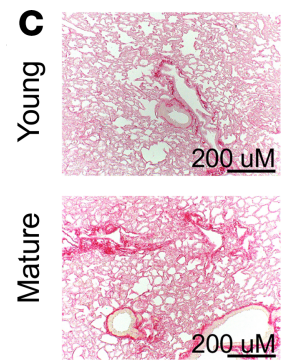

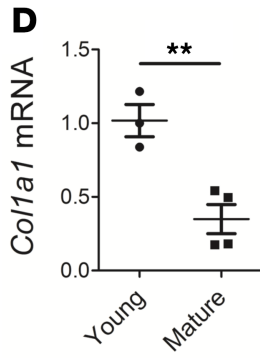

- Young
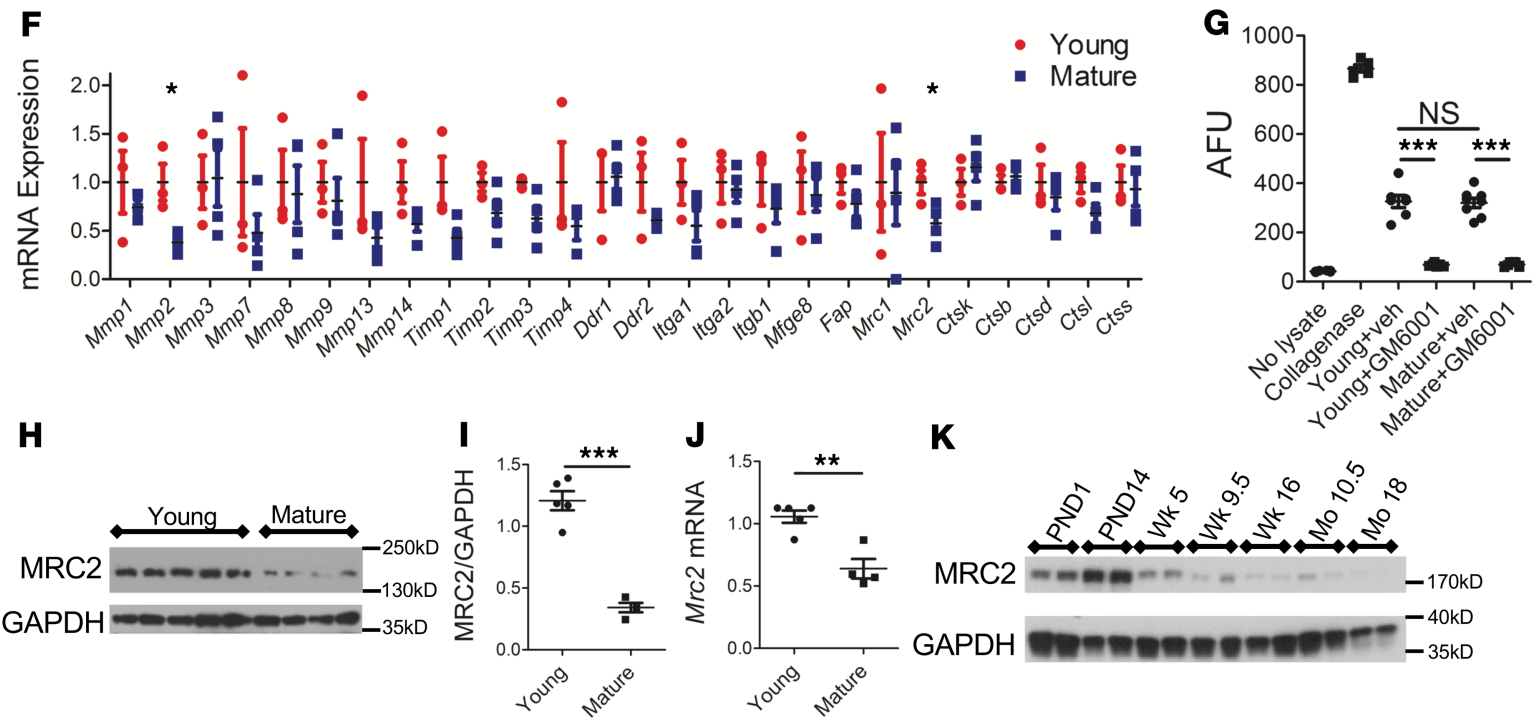

$\mathbf{K}$
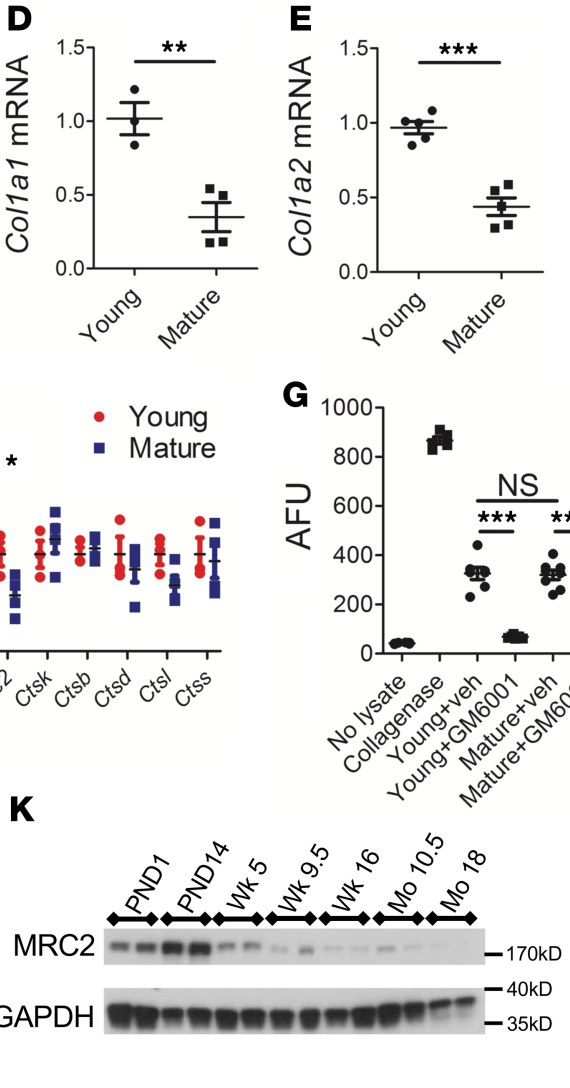

Figure 1. Decreased cell-mediated collagen degradation accompanies an increase in collagen content during maturation. (A) Hydroxyproline content of mouse lung from young versus mature animals; $n=5$ female mice in each group. (B) Hydroxyproline content of mouse lung from young versus mature animals normalized to lung weight; $n=5$ female mice in each group. (C) Representative images of picrosirius red staining of mouse lung (original magnification, $\times 100$ ). ( $\mathbf{D}$ and E) Quantitative real-time PCR (Q-RT-PCR) of Col1a1 (D) and Col1a2 (E) from mouse whole lung; $n=3-5$ male mice in each group. In this and subsequent figures showing Q-RT-PCR, data are normalized to a housekeeping gene by the $2^{-\Delta \Delta C t}$ method and then expressed relative to the initial control condition. (F) Q-RT-PCR of indicated genes in whole mouse lung; $n=3-4$ female mice in each group. (G) DQ-collagen degradation assay. $n=5-6$ for no lysate and collagenase controls, $n=7-8$ for remaining experimental conditions, a mix of male and female in each group; collagenase was used as a positive control. AFU, arbitrary fluorescence units. (H and I) Representative Western blot and densitometry of whole mouse lung for MRC2; $n=4-5$ male mice in each group; GAPDH is a loading control. (J) Q-RT-PCR of Mrc2 in whole mouse lung; $n=4-5$ male mice in each group. (K) Representative Western blot of MRC2 from whole mouse lung at indicated time points ( $P N D$, postnatal day; Wk, week; Mo, month); each lane represents an independent sample; a mix of male and female mice were used. Statistics: (A-F and I-J) Student's $t$ test, (G) ANOVA. ${ }^{*} P<0.05,{ }^{* *} P<0.01,{ }^{* * *} P<0.001$.

We were next interested in whether the diminution of Mrc2 expression had functional consequences in a model of fibrosis. Aging has been shown to impair resolution of fibrosis (6). A single dose of bleomycin induced an increase in collagen content at an early time point (11 days) in both young and mature animals (Figure 3, A and B; given baseline differences in lung collagen and lung weight, young and mature mice were analyzed separately). However, 8 weeks after bleomycin (Figure 3, C-E), only mature mice exhibited an increase in collagen content relative to saline controls, thus indicating an impairment in resolution of fibrosis even in 10- to 12-month-old mice.

It has been previously published that $\mathrm{Mrc}^{-1-}$ mice exhibit increased lung fibrosis 4 weeks after bleomycin administration (22), a time point when in C57BL/6 mice the resolution process has begun (6). Therefore, young $\mathrm{Mrc2}^{-/-}$mice were used to test the hypothesis that $\mathrm{Mrc2}$ deficiency alone is sufficient to impair resolution of fibrosis. We used a single-dose bleomycin fibrosis model and evaluated collagen content at 11 days (Figure $3 \mathrm{~F}$ ) and 8 weeks (Figure 3G). These data show that mice exhibited a similar increase in fibrosis at the early time point, but collagen content was significantly elevated only in the $\mathrm{Mrc2}^{-/-}$animals in the bleomycin group at the late time point. Thus, Mrc2 gene deletion is sufficient to impair resolution of fibrosis.

To investigate ECM dynamics during fibrosis in young versus mature animals, Q-RT-PCR was performed on whole lung after administration of a single dose of bleomycin (Figure 3, H and I). As expected, collagen transcription increased markedly in the bleomycin model in young animals, with a concomitant 
A CD45-PDGFRA+ CD45+F4/80+
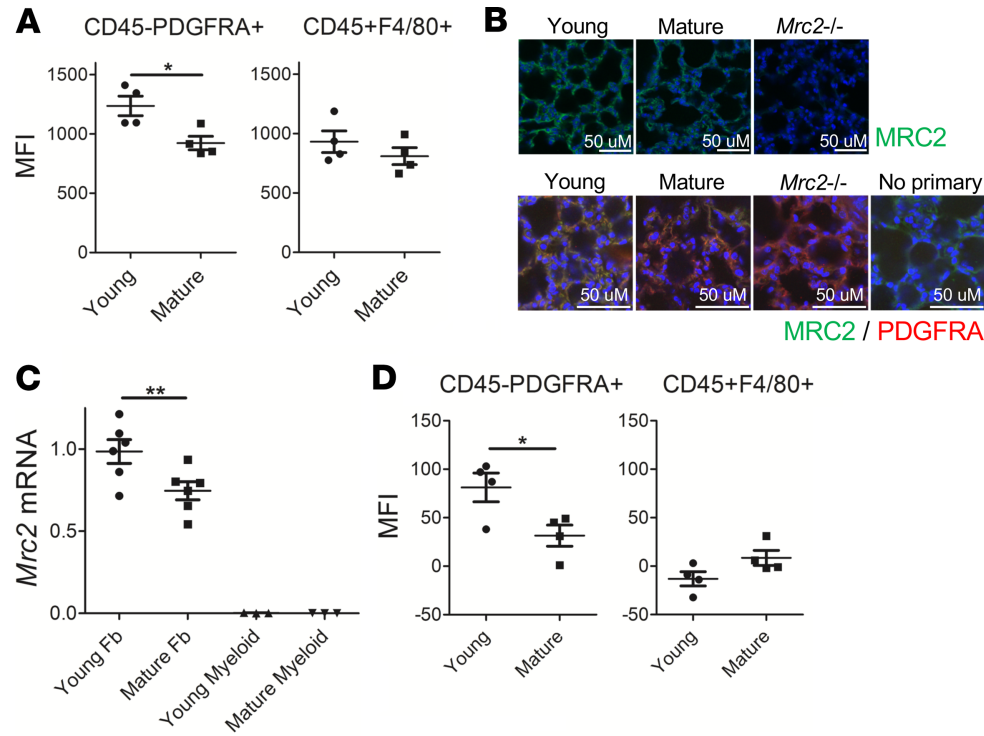

D CD45-PDGFRA+ CD45+F4/80+
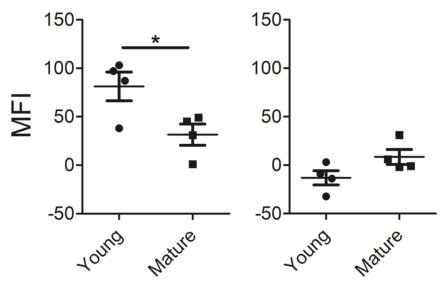

Figure 2. Fibroblasts exhibit decreased Mrc2 expression during maturation. (A) Fluorescent collagen uptake assay in lung cells after gating for markers as indicated; $n=4$ female mice in each group. (B) Representative immunofluorescence staining of tissue sections from WT mouse lungs with sections from $\mathrm{Mrc2}^{-1-}$ mice serving as control; top: original magnification, $\times 400$, with MRC2 in green; bottom: original magnification, 630x, with MRC2 in green, costained with PDCFRA in red; no primary, no PDGFRA antibody; DAPI is a counterstain. (C) Q-RT-PCR in sorted fibroblasts or myeloid cells for Mrc2; $n=3-6$; a mix of male and female mice were used. (D) Flow cytometric staining for MRC2 after gating for markers as indicated; $n=$ 4; a mix of male and female mice were used. Statistics: (A and D) Student's $t$ test, (C) ANOVA. ${ }^{*} P<0.05,{ }^{* *} P<0.01$.

modest increase in Mrc2 expression. In contrast, collagen transcription was induced to a lesser extent after bleomycin in mature animals, with a nonsignificant increase in $\mathrm{Mrc2}$ expression. These data further indicate that impaired resolution of fibrosis in mature animals may not be primarily due to excessive collagen production but rather failure of collagen degradation.

Because age-dependent Mrc2 downregulation occurs at the transcript level, we set out to identify transcriptional regulators of $M r c 2$ expression. A previous study from our laboratory identified an ortholog of myeloid zinc finger-1 (Mzf1) as regulating collagen uptake in Drosophila phagocytes $(19,23)$. The presence of multiple informatics-predicted candidate binding sites for MZF1 in the putative promoter region of Mrc2 suggested that this may be a transcription factor for Mrc2 (Figure 4A). Furthermore, publicly available ChIP-Seq data demonstrate a strong peak in the same region of the human $M R C 2$ locus upstream of the transcriptional start site (Figure 4B).

Therefore, a cell culture system was used to test whether MZF1 controls MRC2 expression. ShRNA-mediated silencing of $M Z F 1$ led to decreased collagen uptake in an in vitro collagen uptake assay (Figure 4C and Supplemental Figure 5A). In addition, silencing of MZF1 led to decreased MRC2 protein (Figure 4D) and $M R C 2$ transcript (Figure 4E). These data are consistent with positive regulation of $M R C 2$ by MZF1. To confirm that MZF1 can drive transcription from this region, a 625-bp region of the putative promoter locus of $M R C 2$ (corresponding to the ChIP-Seq peak) was subcloned into a luciferase vector, and a standard luciferase assay was used to show that luciferase activity downstream of this region occurs in response to $M Z F 1$ cotransfection but not when candidate binding sites are mutated or if a BFP control vector is used instead of $M Z F 1$ (Figure 4, F and G; and Supplemental Figure 5, B-D). Finally, MZF1 protein levels were measured by Western blot in young and mature mouse lung tissue at baseline or after bleomycin (Figure 4H). This demonstrated decreased MZF1 protein levels in mature lungs corresponding to diminished MRC2 protein levels, and in 2 out of 4 samples, there was a relative increase in MZF1 protein levels after bleomycin corresponding to increased MRC2 protein levels. Of note, though human MZF1 is commonly seen at $82 \mathrm{kDa}$, a SUMOylated form that is higher molecular weight $(\sim 100 \mathrm{kDa})$ has been well described (24); this was the dominant band in mouse tissue and thus is the band that is shown in blots of mouse tissue lysates. Therefore, these studies identified MZF1 as a potentially novel transcription factor that positively regulates $M R C 2$ expression, exhibits occupancy in the $M R C 2$ promoter region, and can drive transcription off the region upstream of the $M R C 2$ transcriptional start site. 

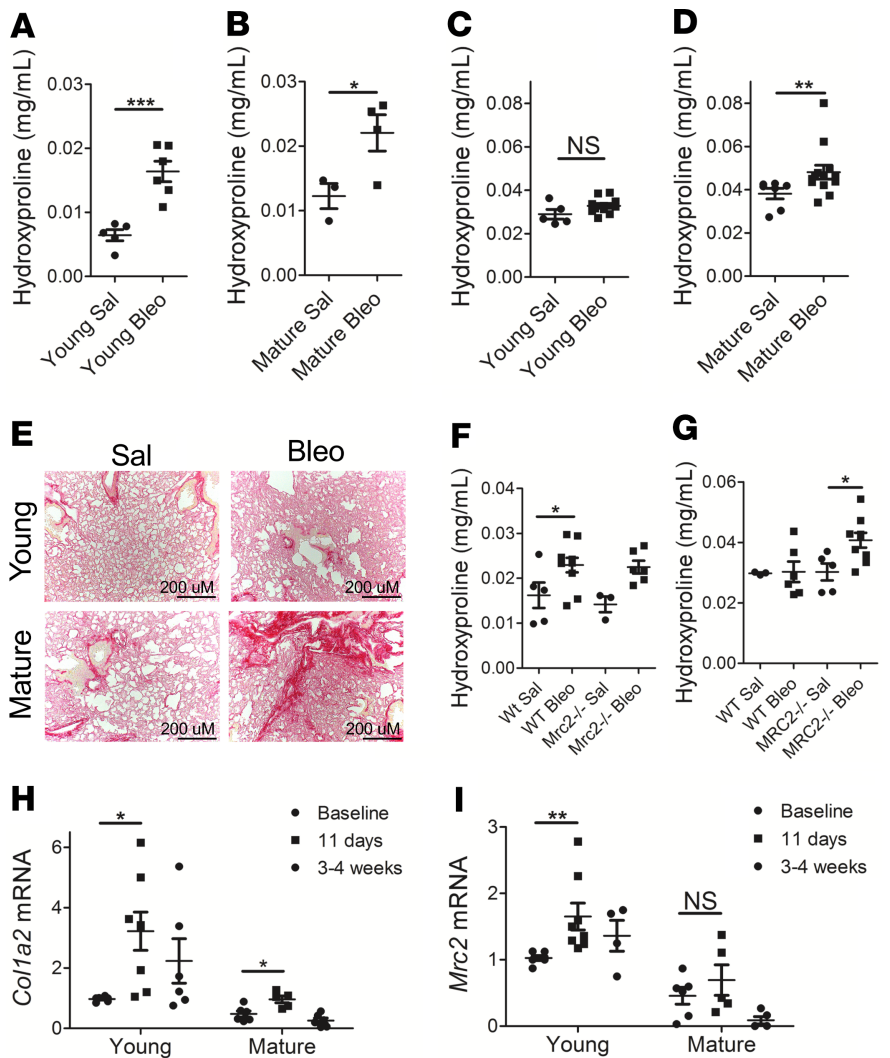

Figure 3. Impaired resolution of bleomycin-induced lung fibrosis in mature mice. (A-D) Colorimetric quantification of lung hydroxyproline content in mature or young animals 11 days ( $\mathbf{A}$ and

B) or 8 weeks (C and $\mathbf{D})$ after bleomycin or saline administration; $n$ = 3-13; a mix of male and female mice were used. (E) Representative images of picrosirius red staining of lungs from young and mature mice 8 weeks after saline or bleomycin administration at original magnification, $\times 100$. (F and $\mathbf{G}$ ) Lung hydroxyproline content in WT or Mrc2 ${ }^{-/-}$animals 11 days (F) or 8 weeks (G) after bleomycin or saline administration; $n=3-10$; a mix of male and female mice were used. ( $\mathbf{H}$ and $\mathbf{I})$ Q-RT-PCR for Col1a2 (H) or Mrc2 (I) from whole lung at baseline or at indicated time points after bleomycin administration in young or mature animals; $n=4-8$; a mix of male and female mice were used. Statistics: (A-C) Student's $t$ test, (D) Mann-Whitney $U$ test, (F-H) ANOVA, (I) Kruskal-Wallis test. ${ }^{*} P$ $<0.05,{ }^{* *} P<0.01,{ }^{* * *} P<0.001$.

We next sought to determine whether the age-dependent downregulation of MZF1 and MRC2 occurred in other tissues. Immunoblot analysis of both mouse heart and skin was performed to evaluate protein expression levels of these molecules (Figure 5, A and B). This analysis demonstrated similar age-dependent downregulation of MRC2 and MZF1 protein levels. Immunofluorescence staining of skin also showed marked downregulation of MRC2 protein levels in dermal cells with age (Figure 5C). Therefore 3 organs demonstrated age-dependent downregulation of MRC2 with a corresponding decrease in MZF1 protein levels.

Finally, we investigated human specimens. MRC2 levels were measured by Q-RT-PCR in whole lung tissue obtained from human lungs deemed not suitable for organ donation (Figure 6, A and B), and collagen uptake assays were done using normal human lung fibroblasts obtained from biopsy or postmortem specimens (Figure 6, C and D). These 2 experiments showed age-dependent downregulation of $M R C 2$ in whole human lung and decreased collagen uptake in human lung fibroblasts.

\section{Discussion}

The work we have presented in this manuscript shows that collagen turnover decreases during the life span of mice and that this decrease is marked by both decreased collagen transcription and cell-mediated collagen internalization and degradation by MRC2. These dynamics are also found in bleomycininduced fibrosis, in which the impaired resolution of mature animals is marked primarily by decreased collagen turnover rather than excessive collagen synthesis. We have characterized $M z f 1$ as a novel transcription factor for Mrc2 that may play an important role in these age-dependent findings. What is more, these gene expression changes extend beyond the lung, at least to heart and skin. Finally, we demonstrated analogous findings in human lung. Intriguingly, a synthesis of prior literature shows that in human skin, collagen production decreases with age (25), but total collagen content does not change (26). This discrepancy implies a significant age-dependent reduction in collagen turnover in human skin as well. Therefore, we believe we have described an aspect of ECM homeostasis that is conserved among both different organs and divergent mammalian species.

The "mature" animals we used in our study are not as old as those in many studies of aging. Nonetheless, our data show that Mrc2 decreases as mice mature into adulthood. Mice even at 10-12 months 

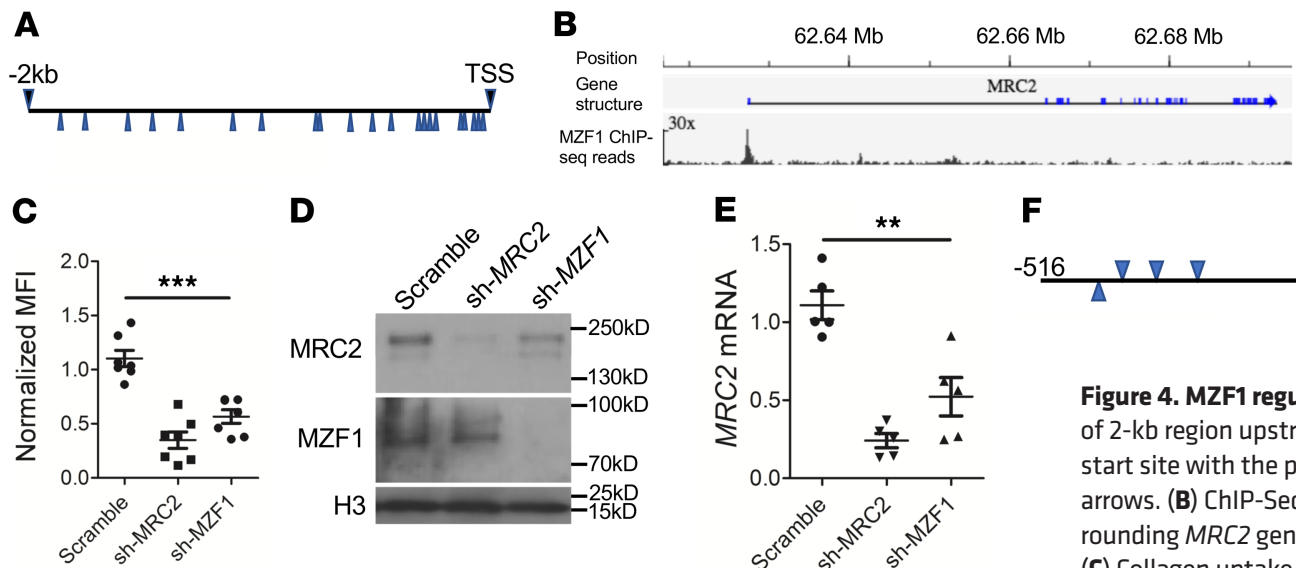
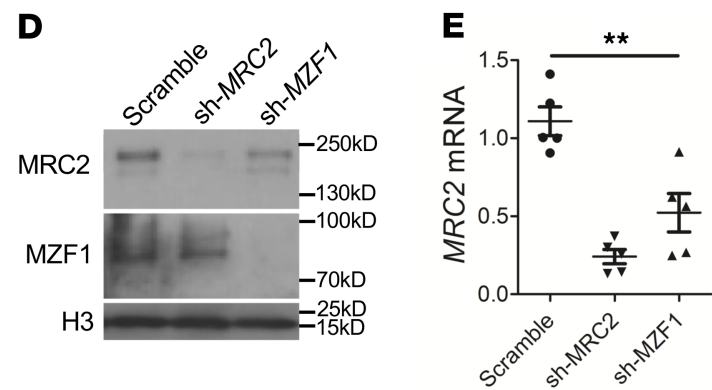

$\mathbf{F}$

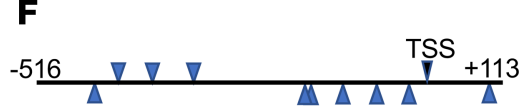

Figure 4. MZF1 regulates MRC2 expression. (A) Schematic of 2-kb region upstream of the human $M R C 2$ transcriptional start site with the putative MZF1 binding sites marked by blue arrows. (B) ChIP-Seq data from Encode ENCSR298QUH surrounding MRC2 gene. Scale bar: original magnification, $\times 30$. (C) Collagen uptake assay in U937 cells treated with shRNA targeting MZF1 (sh-MZF1) or MRC2 or a scrambled control; $N$ = 7. (D) Western blot in U937 cells treated with sh-MZF1 versus scramble or sh-MRC2 control against MRC2 and MZF1; histone $\mathrm{H} 3$ is a loading control (representative of $N=2$ ). (E) Q-RTPCR for MRC2 in sh-MZF1 cells versus scramble or sh-MRC2 control; $N=5$. (F) Schematic of MZF1 putative binding sites (blue arrows) corresponding to the 625-bp peak noted in B. (G) Luciferase activity in U2OS cells treated with luciferase vector with the 625 bp from $\mathbf{F}$ or same sequence with 2-bp mutations in MZF1 binding sites (mut-MRC2) as well as MZF1 overexpression vector or $B F P$ control; $N=4$. (H) Representative Western blot for MRC2 and MZF1 of whole-lung lysates from young or mature mice 11 days after treatment with bleomycin or saline control; $n=3-4$; a mix of male and female mice were used; GAPDH is a loading control. Statistics: (C, E, G) ANOVA. ${ }^{*} P<$ $0.05,{ }^{* *} P<0.01,{ }^{* * *} P<0.001$.

of age demonstrate impaired resolution of fibrosis in a bleomycin model compared with young animals. These findings may thus be applicable to aging during periods even before the onset of advanced age and are not confounded by comorbid conditions associated with advanced aging. From a therapeutic standpoint, understanding mechanisms at earlier stages of aging may allow interventions to be taken when diseases are more remediable.

An ability to restore levels of collagen turnover to those observed in younger individuals may serve as a promising path toward ameliorating fibrotic disease. Though the biology underlying fibrotic disease is complex and involves overproduction of collagen and other matrix proteins as well as dysfunction of multiple other cell types and molecular pathways, a role for impaired matrix degradation may be equally important. Our work also suggests one explanation for some of the difficulty in translating knowledge about the bleomycin fibrosis model into idiopathic pulmonary fibrosis (IPF). If this model is used in young animals, the excess collagen production aspect of the model dominates, whereas in older animals impaired collagen degradation may be more relevant. Such differences could lead to vastly different interpretations about mechanisms of fibrosis and therapeutic efficacy of pharmacologic interventions. Because IPF does not spontaneously resolve in humans, we believe that bleomycin fibrosis in aged animals is a more relevant disease model.

A prima facie conclusion from our work is that ECM synthesis and degradation are linked. The extent to which synthesis and degradation of ECM are directly regulated in tandem or even by the same molecules or upstream events deserves future study. The findings in this study imply that what is desirable is not low ECM production or high ECM degradation but instead a flexible, high-turnover state (i.e., high production and high degradation). This would most accurately describe the state of the developing or immature ECM, i.e., that ECM which is most resistant to impairment in fibrosis resolution. Other questions raised by this study are what cues during maturation lead to downregulation of MZF1 expression and what signals induce MZF1 after bleomycin injury. Teleologically, one might conjecture that diminished collagen turnover during aging represents a trade-off between a flexible ECM and other diseases of aging, such as cancer, in which a flexible ECM may be maladaptive for the host. 
A
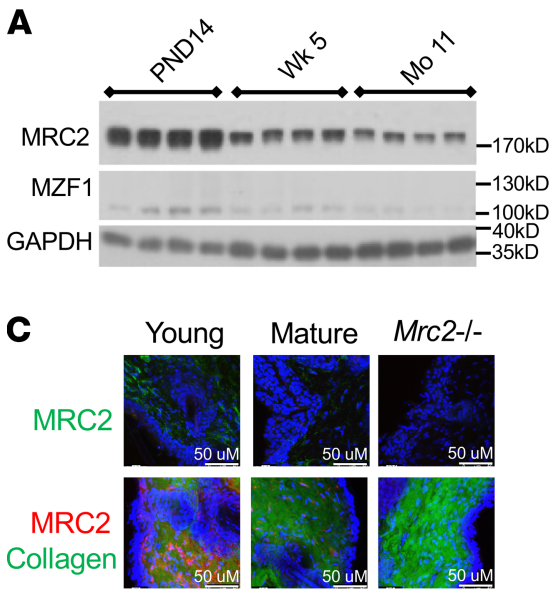

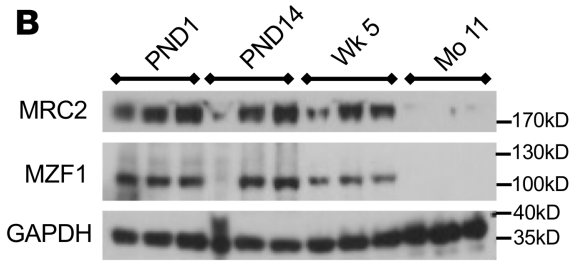

Figure 5. Age-dependent downregulation of MRC2 and MZF1 downregulation in other tissues. (A and $\mathbf{B}$ ) Representative Western blot of MRC2 in whole mouse heart (A) or skin (B) lysates at different time points as indicated for MRC2 and MZF1; GAPDH is a loading control; $n=3-4$ female mice per group. (C) Representative immunofluorescence images (original magnification, $\times 400$ ) of skin tissue sections from young or mature animals with tissue sections from $\mathrm{MrC2}^{-1-}$ mice serving as control; top: MRC2 in green; bottom, GFP-collagen mice, with MRC2 in red; DAPI is a counterstain.

Recent literature demonstrating that MZF1 positively regulates other aspects of matrix degradation in cancer, including cathepsin expression (27) and lysosomal function (24), is consistent with this concept. Or, perhaps retaining a flexible ECM after maturation is simply energetically wasteful. These remain worthy questions that we hope to address in future work.

\section{Methods}

Mice. All WT mice for young versus mature experiments were C57BL/6 and used at 5-8 weeks (young) or 10-12 months (mature). $\mathrm{Mrc}^{-1-}$ mice were generated by T.H. Bugge et al. as previously described by deletion of exons 2-6, which results in a truncated transcript and protein that is lacking the collagenbinding domain (15). GFP-collagen-transgenic mice used in Figure 4 were generated as previously described by inserting a GFPtopaz tag into the mouse pro- $\alpha 2$ (I) collagen N-terminus under control of the 3.6-kb type I collagen promoter (28). These transgenic mice were generated on a C57BL/6N background. All experimental protocols were approved by the UCSF IACUC for animal studies.

Hydroxyproline. Whole left mouse lung was dissected, homogenized, precipitated with trichloroacetic acid, and baked overnight at $110^{\circ} \mathrm{C}$ in concentrated $\mathrm{HCl}(12 \mathrm{~N})$ to yield total dry hydrolyzed protein. Total hydrolyzed protein samples were vigorously resuspended in pure water via orbital shaker for 2 hours. Chloramine-T $(0.06 \mathrm{M})$ was added and samples were incubated at room temperature for 20 minutes; Ehrlich's solution, 30\% perchloric acid and 4-(Dimethylamino)benzaldehyde (1 M) in 2-propanol, was added, and

A

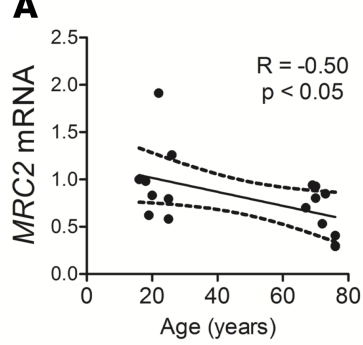

C

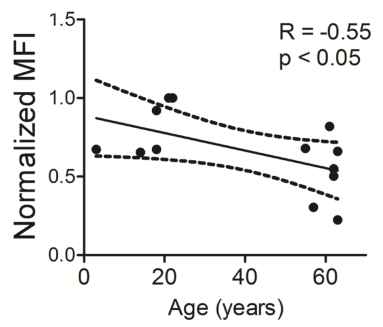

B

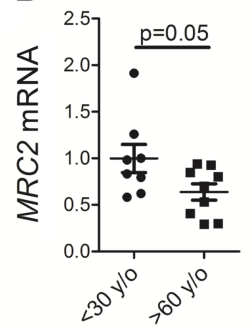

D

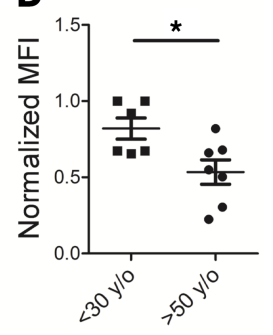

Figure 6. Human lung also exhibits age-dependent downregulation of MRC2 and cell-mediated collagen uptake in fibroblasts. (A and B) Q-RT-PCR of wholelung lysates from humans of different ages for $M R C 2$ is displayed according to age (A) or by group (B); $N=17$ total, 8-9 per group. (C and D) Collagen uptake from human lung fibroblasts from humans of different ages is displayed according to age (C) or by group (D); $N=13$ total, 6-7 per group. Statistics: (A and C) Pearson's correlation with linear correlation shown as well as $95 \%$ confidence interval (dotted lines); ( $B$ and D) Student's $t$ test. ${ }^{*} P<0.05$. 
samples were incubated for 15 minutes at $60^{\circ} \mathrm{C}$. Hydroxyproline content was then spectrophotometrically quantified by measuring sample absorbance at $550 \mathrm{~nm}$ relative to standards produced from solutions of known hydroxyproline (MilliporeSigma) concentration.

Picrosirius red staining. Inferior lobes of the mouse right lung were dissected from young and mature mice and inflated with $4 \%$ paraformaldehyde in PBS and incubated overnight at $4^{\circ} \mathrm{C}$. The lobes were washed twice with PBS and stored in 70\% ethanol before paraffin embedding, sectioning (5- $\mu \mathrm{m}$ thickness), and staining with picrosirius red for evaluation of fibrosis as previously described $(29,30)$.

Q-RT-PCR. Total RNA from tissue or sorted cells was isolated via RNeasy Mini Kit (QIAGEN). Total RNA from each sample was reverse-transcribed with a first-strand cDNA synthesis kit (Quantabio) according to the manufacturer's instructions. Q-RT-PCR reactions were performed with different sets of primers and Sensifast SYBR (Bioline) on a CFX384 Real-Time PCR Detection System (Bio-Rad) using $20 \mathrm{ng}$ cDNA/reaction. Each Q-RT-PCR reaction was performed at least twice, and representative results are shown relative to a control by the standard $2^{-\Delta \Delta \mathrm{Ct}}$ method, where $\mathrm{Ct}$ represents the number of cycles required to reach threshold for the target gene subtracted from the number of cycles required to reach threshold for a control housekeeping gene (Gapdh or Actb). All data are shown relative to the first control condition in each figure panel. Primer sequences are in the Supplemental Methods.

$D Q$-collagen degradation assay. In total, $10 \mu \mathrm{g}$ of total lung homogenates (made in $150 \mathrm{mM} \mathrm{NaCl}, 50 \mathrm{mM}$ Tris- $\mathrm{HCl} \mathrm{pH} 8.0,1 \%$ Nonidet P-40) from young and mature mice were plated with PBS in opaque 96-well black plates in duplicate and incubated at $37^{\circ} \mathrm{C}$ with $10 \mu \mathrm{g} / \mathrm{mL}$ DQ-collagen I (Invitrogen, Thermo Fisher Scientific), a dye-conjugated substrate that fluoresces upon proteolytic degradation of collagen molecules. Plates were protected from light during incubation. Fluorescence was quantified via spectrofluorometer (Molecular Devices Spectramax M2 65) after 24 hours at absorption and emission maxima of $485 \mathrm{~nm}$ and $538 \mathrm{~nm}$, respectively. Mean fluorescence intensity is shown in which each data point displayed represents the mean of 2 technical replicates for an individual mouse.

Immunoblot. Cells or tissues were homogenized with cold lysis buffer $(50 \mathrm{mM}$ Tris- $\mathrm{HCl} \mathrm{pH} 7.5,150$ $\mathrm{mM} \mathrm{NaCl}, 5 \mathrm{mM}$ EDTA, $0.1 \%$ SDS, and 1\% Nonidet P-40) supplemented with protease and phosphatase inhibitor cocktail (Thermo Fisher Scientific). Lysates were cleared by centrifugation at 15,000 $g$ for 15 minutes at $4^{\circ} \mathrm{C}$, electrophoresed under reducing conditions on $10 \%$ precast gels (Bio-Rad), and transferred to PVDF membranes (Immobilon-P). Membranes were blocked for 1 hour in $5 \%$ bovine serum albumin and incubated with primary antibodies against MRC2 (1:800; R\&D Systems, Bio-Techne; anti-human: AF5770; anti-mouse: AF4789), MRC1 (1:800; R\&D Systems, Bio-Techne, AF2534), MZF1 (1:1000; antimouse: ABclonal Technology, A10356; anti-human: Abcam, ab64866), GAPDH (1:10,000; Cell Signaling Technology, 14C10), or histone H3 (1:10,000; Cell Signaling Technology, D1H2) overnight at $4^{\circ} \mathrm{C}$. Membranes were then incubated with horseradish peroxidase-conjugated secondary antibodies (1:5000; Cell Signaling Technology; Santa Cruz Biotechnology) for 1 hour at room temperature. Blots were developed using enhanced chemiluminescence (GE Healthcare Life Sciences) by exposure onto autoradiography film. Where indicated densitometry was performed using Image $(\mathrm{NIH})$, normalizing the protein of interest to a loading control (GAPDH), and comparisons were made to the baseline condition.

Flow cytometry and flow cytometry-based collagen uptake assay. Flow cytometric analysis was done using a FACSVerse (BD Biosciences), a fluorescence-activated cell analyzer, and sorting was done using FACSAria II or FACSAria III (BD Biosciences) fluorescence-activated cell sorters. For primary cells, cells were isolated by homogenizing whole lung via mechanical dissociation followed by incubation with a cocktail of collagenase (Roche), dispase (Roche) and DNase I (MilliporeSigma). For the collagen uptake assay, cells were then washed and incubated in fresh DMEM with Oregon Green 488 -conjugated gelatin $(10 \mu \mathrm{g} / \mathrm{mL}$ final concentration; Life Technologies, Thermo Fisher Scientific) for 1 hour at $37^{\circ} \mathrm{C}$. Cells were then trypsinized and stained using a standard FACS staining protocol. Antibodies included anti-CD45, -PDGFRA, and -F4/80 (BioLegend); for MRC2, the sheep anti-mouse antibody (above) was used as a primary antibody with a secondary of donkey anti-sheep conjugated to a fluorophore (A-21448, Invitrogen, Thermo Fisher Scientific). Cells were then analyzed or sorted on one of the above devices, with sorting and analysis gates based on fluorescence-minus-one controls after compensation was performed with single-stain controls. For MRC2 staining experiments, average fluorescence from $\mathrm{Mrc}^{-/-}$samples was subtracted from all experimental samples to eliminate background fluorescence. For the collagen uptake assay in Figure $3 \mathrm{C}$ and Figure 4, F and G, U937 cells (ATCC) were washed and resuspended in fresh RPMI medium, or primary fibroblasts were washed and incubated with fresh DMEM, with Oregon Green 488-conjugated gelatin 
(10 $\mu \mathrm{g} / \mathrm{mL}$ final concentration; Life Technologies, Thermo Fisher Scientific) for 1 hour at $37^{\circ} \mathrm{C}$ followed by washing and flow cytometric analysis. Data were analyzed with FlowJo, version 7.6.1 (Tree Star).

Immunofluorescence. Right lung lobes or shaved full thickness back skin were fixed in 4\% paraformaldehyde in PBS and then dehydrated in sucrose, embedded in OCT, and frozen in chilled isopropanol on dry ice. Frozen sections $(50 \mu \mathrm{m})$ were prepared. Sections were blocked in PBS with $0.5 \%$ bovine serum albumin, $0.1 \%$ Triton X-100, and 5\% donkey serum. Antibodies against MRC2 (R\&D Systems, Bio-Techne, as above) and/or PDGFRA (rat anti-mouse, clone APA5, BD Biosciences) were applied at 1:100 dilution followed by secondary antibodies conjugated to Alexa Fluor 488 or 594 (Invitrogen, Thermo Fisher Scientific A-11015 and A-11016 or Abcam ab150155) at 1:100. Sections were mounted in VECTASHIELD (Vector Laboratories). Images were captured using a Leica TCS SPE confocal microscope with an ACS APO $40 \times$ or $63 \times$ oil CS objective lens at room temperature with Leica Type F Immersion Liquid (catalog 11513859). Images were processed with ImageJ, in which brightness/contrast was changed uniformly across all conditions and pseudocoloring was applied. Single optical slices at Airy 1 are shown for the lung to highlight lung architecture, whereas average $Z$-projections through a $Z$-stack of the entire tissue slice are shown for the skin.

Bleomycin model of pulmonary fibrosis. To induce experimental lung fibrosis, mice were intratracheally challenged with a single dose of 1.5-2.5 U/kg bleomycin sulfate diluted in PBS or vehicle control (the dose was the same for every mouse within each experiment; Selleck Chemicals) after induction of general anesthesia with isoflurane using a precision vaporizer. Body mass was regularly measured for 14 days postinstillation. Mice were euthanized at time points as indicated in the text, and lungs were harvested for downstream analysis.

Cell culture, shRNA, and luciferase experiments. For validation of the MRC2 antibody for flow cytometry, HEK293T (ATCC) cells were grown in DMEM (Corning) with 10\% FBS (Gemini Bio-Products) and transfected with pCI-Mrc2 (murine Mrc2 subcloned into the pCI-Neo expression vector, from Promega) using Lipofectamine 2000 (Invitrogen, Thermo Fisher Scientific) according to the manufacturer's instructions. For the MZF1 experiments, human U937 (acquired from ATCC) were grown in RPMI1640 with L-glutamine (Corning) with 10\% heat-inactivated FBS (Gemini Bio-Products). Lentiviral particles harboring shRNA machinery within pSicoR-puromycin vectors were obtained from MilliporeSigma (scramble control: SHC002V; sh-MZF1: CCGGCCGCAGGTCCAGGTAGTGTAACTCGAGTTACACTACCTGGACCTGCGGTTTTT; sh-MRC2: CCGGCCGGTATTGCTATAAGGTGTTCTCGAGAACACCTTATAGCAATACCGGTTTTT). Lentiviral infection at 10 multiplicity of infection was performed in cells overnight in complete medium after addition of polybrene, and medium was replaced with fresh complete medium the next day. The infected cells were allowed to grow and then selected by resistance to puromycin ( $1 \mu \mathrm{g} / \mathrm{mL}$ for U937; MilliporeSigma). Puromycin-selected cells were collected for Q-RT-PCR, collagen uptake, and Western blotting. Cells were infected at passages 3-6, and cells were collected for experiments between passages 5 and 16. For the luciferase assay, a 625-bp fragment around the $M R C 2$ transcriptional start site as shown, or the same fragment with 2 A-T substitutions within each of the 11 MZF1 predicted binding sites, was subcloned into the multiple cloning site in a firefly luciferase vector with a minimal promoter element (Promega). Using Lipofectamine 2000 according to the manufacturer's instructions, this was transfected into U2OS cells (ATCC) along with a constitutive renilla luciferase vector (Promega) and a $\mathrm{pEBFP}$ or $\mathrm{pEBFP}-M Z F 1$ vector, which were previously described and gifts of T. Sander (Medical College of Wisconsin, Milwaukee, Wisconsin, USA) (31). The Stop\&Glo (Promega) system was used to read luciferase activity in cells on a luminometer (Promega GloMax). All firefly luciferase values were normalized to renilla luciferase levels for a transfection control, and then data were displayed relative to transfection with the $\mathrm{pEBFP}$ (empty vector) with the endogenous promoter sequence firefly luciferase construct.

Human lung tissue and primary human lung fibroblast isolation. Normal human lung tissue from donors of different ages was obtained from lungs not used by the Northern California Transplant Donor Network according to an IRB-exempted protocol (UCSF). After harvest, lung tissue was directly snap-frozen in liquid nitrogen and RNA was extracted as above. Primary human lung fibroblasts from subjects of different ages were obtained from 2 laboratories (UCSF and University of Michigan). Normal donor lungs were provided to the University of Michigan by Gift of Life Michigan. Adult lung parenchyma was collected from lobectomy specimens from resections performed on primary lung cancer (UCSF) or from normal lungs not used for transplantation (UCSF and University of Michigan). Nonfibrotic lung tissue was verified as "normal" by histology and further confirmed by lack of alternative diagnoses and normal pulmonary function studies in subjects when data were available. Fibroblasts were cultured from outgrowths of minced lung tissue. Informed consent was obtained from all surgical participants as part 
of an approved ongoing research protocol by the UCSF Committee on Human Research in full accordance with the Declaration of Helsinki principles. Specimens from deceased donors were IRB exempted. Fibroblasts were thawed and grown in DMEM (Corning) with 10\% FBS (Gemini Bio-Products) supplemented with pen/strep (Gibco, Thermo Fisher Scientific) and then used for the collagen uptake assay. Fibroblasts were used only at early passage $(<3-4$ passages).

Statistics. Data were evaluated with GraphPad Prism software: for 2-sample comparisons, by 2-tailed Student's $t$ test or Mann-Whitney $U$ test if data did not pass a test of normality; for multiple comparisons, by 1-way ANOVA with post hoc Bonferroni's testing if the ANOVA was significant or Kruskal-Wallis test with post hoc Dunn testing if the Kruskal-Wallis test was significant if data did not pass a test of normality; or by Pearson's correlation as indicated. A $P$ value of 0.05 or less was considered statistically significant with ${ }^{*} P<0.05,{ }^{* *} P<0.01,{ }^{* *} P<0.001$, and NS $=$ not significant. Data are presented as mean \pm SEM.

Study approval. All experiments using mice were approved by the Institutional Animal Care and Use Committee of UCSF. All experiments using human tissue were approved by the UCSF or University of Michigan Committee on Human Research. Informed consent was obtained for study participants or waived for human tissue from deceased donors in accordance with the UCSF or University of Michigan Committee on Human Research.

\section{Author contributions}

MJP designed and performed the experiments, analyzed the data, and wrote the manuscript. CDY aided in design and performance of the experiments, except for some Q-RT-PCR experiments and picrosirius red staining, which were done by CJLS. RD and CLV aided in the design and interpretation of results. SLN and SKH provided human fibroblasts, and PJW provided human whole lung tissue RNA. SLD provided GFP-collagen mice. KA aided in design of the study, analysis of the data, and writing of the manuscript. All authors contributed to editing the manuscript.

\section{Acknowledgments}

This research was supported by US NIH grants K08 HL145015 (to MJP) and R01 HL136377 (to KA), as well as an American Thoracic Society Foundation/American Lung Association Research Grant (to MJP). We would like to thank Tara Sander for providing MZF1 constructs and Ryan Ta for help with mouse husbandry and Western blotting. We would also like to thank S. Layer for ongoing inspiration.

1. Raghu G, Weycker D, Edelsberg J, Bradford WZ, Oster G. Incidence and prevalence of idiopathic pulmonary fibrosis. Am J Respir Crit Care Med. 2006;174(7):810-816.

2. Armanios MY, et al. Telomerase mutations in families with idiopathic pulmonary fibrosis. NEngl J Med. 2007;356(13):1317-1326.

3. Gulati S, Thannickal VJ. The aging lung and idiopathic pulmonary fibrosis. Am J Med Sci. 2019;357(5):384-389.

4. Lorenz HP, Whitby DJ, Longaker MT, Adzick NS. Fetal wound healing. The ontogeny of scar formation in the non-human primate. Ann Surg. 1993;217(4):391-396.

5. Stern M, Dodson TB, Longaker MT, Lorenz HP, Harrison MR, Kaban LB. Fetal cleft lip repair in lambs: histologic characteristics of the healing wound. Int J Oral Maxillofac Surg. 1993;22(6):371-374.

6. Hecker L, et al. Reversal of persistent fibrosis in aging by targeting Nox4-Nrf2 redox imbalance. Sci Transl Med. 2014;6(231):231ra47.

7. Spagnolo P, Maher TM, Richeldi L. Idiopathic pulmonary fibrosis: recent advances on pharmacological therapy. Pharmacol Ther. 2015;152:18-27.

8. Mays PK, Bishop JE, Laurent GJ. Age-related changes in the proportion of types I and III collagen. Mech Ageing Dev. 1988;45(3):203-212

9. Takubo Y, Hirai T, Muro S, Kogishi K, Hosokawa M, Mishima M. Age-associated changes in elastin and collagen content and the proportion of types I and III collagen in the lungs of mice. Exp Gerontol. 1999;34(3):353-364.

10. Bradley KH, McConnell SD, Crystal RG. Lung collagen composition and synthesis. Characterization and changes with age. J Biol Chem. 1974;249(9):2674-2683.

11. McKleroy W, Lee TH, Atabai K. Always cleave up your mess: targeting collagen degradation to treat tissue fibrosis. Am JPhysiol Lung Cell Mol Physiol. 2013;304(11):L709-L721.

12. Tabula Muris Consortium, et al. Single-cell transcriptomics of 20 mouse organs creates a Tabula Muris. Nature. 2018;562(7727):367-372.

13. Atabai K, et al. Mfge8 diminishes the severity of tissue fibrosis in mice by binding and targeting collagen for uptake by macrophages. J Clin Invest. 2009;119(12):3713-3722.

14. Messaritou G, East L, Roghi C, Isacke CM, Yarwood H. Membrane type-1 matrix metalloproteinase activity is regulated by the endocytic collagen receptor Endo180. J Cell Sci. 2009;122(pt 22):4042-4048.

15. Engelholm LH, et al. uPARAP/Endo180 is essential for cellular uptake of collagen and promotes fibroblast collagen adhesion. J Cell Biol. 2003;160(7):1009-1015. 
16. Zhang J, Salamonsen LA. In vivo evidence for active matrix metalloproteinases in human endometrium supports their role in tissue breakdown at menstruation. J Clin Endocrinol Metab. 2002;87(5):2346-2351.

17. Howard MJ, Isacke CM. The C-type lectin receptor Endo180 displays internalization and recycling properties distinct from other members of the mannose receptor family. J Biol Chem. 2002;277(35):32320-32331.

18. Schaum N, et al. The murine transcriptome reveals global aging nodes with organ-specific phase and amplitude. bioRxiv. https://www.biorxiv.org/content/10.1101/662254v1. Published June 7, 2009. Accessed May 1, 2020.

19. Lee TH, et al. Functional genomic screen identifies novel mediators of collagen uptake. Mol Biol Cell. 2014;25(5):583-593.

20. Madsen DH, et al. Tumor-associated macrophages derived from circulating inflammatory monocytes degrade collagen through cellular uptake. Cell Rep. 2017;21(13):3662-3671.

21. Tighe RM, et al. Improving the quality and reproducibility of flow cytometry in the lung. An official American Thoracic Society Workshop report. Am J Respir Cell Mol Biol. 2019;61(2):150-161.

22. Bundesmann MM, Wagner TE, Chow YH, Altemeier WA, Steinbach T, Schnapp LM. Role of urokinase plasminogen activator receptor-associated protein in mouse lung. Am J Respir Cell Mol Biol. 2012;46(2):233-239.

23. Podolsky MJ, et al. Cell division cycle 7 kinase is a negative regulator of cell-mediated collagen degradation. Am J Physiol Lung Cell Mol Physiol. 2018;315(3):L360-L370.

24. Brix DM, et al. Release of transcriptional repression via ErbB2-induced, SUMO-directed phosphorylation of myeloid zinc finger-1 serine 27 activates lysosome redistribution and invasion. Oncogene. 2019;38(17):3170-3184.

25. Varani J, et al. Decreased collagen production in chronologically aged skin: roles of age-dependent alteration in fibroblast function and defective mechanical stimulation. Am J Pathol. 2006;168(6):1861-1868.

26. Lovell CR, Smolenski KA, Duance VC, Light ND, Young S, Dyson M. Type I and III collagen content and fibre distribution in normal human skin during ageing. Br J Dermatol. 1987;117(4):419-428.

27. Rafn B, et al. ErbB2-driven breast cancer cell invasion depends on a complex signaling network activating myeloid zinc finger-1-dependent cathepsin B expression. Mol Cell. 2012;45(6):764-776.

28. Kamel-ElSayed SA, Tiede-Lewis LM, Lu Y, Veno PA, Dallas SL. Novel approaches for two and three dimensional multiplexed imaging of osteocytes. Bone. 2015;76:129-140

29. Sweat F, Puchtler H, Rosenthal SI. Sirius red F3Ba as a stain for connective tissue. Arch Pathol. 1964;78:69-72.

30. Junqueira LC, Bignolas G, Brentani RR. Picrosirius staining plus polarization microscopy, a specific method for collagen detection in tissue sections. Histochem J. 1979;11(4):447-455.

31. Noll L, Peterson FC, Hayes PL, Volkman BF, Sander T. Heterodimer formation of the myeloid zinc finger 1 SCAN domain and association with promyelocytic leukemia nuclear bodies. Leuk Res. 2008;32(10):1582-1592. 